\title{
Fuzzy Time Series Forecasting Model of Inverse Fuzzy Number Based on Percentage Year by Year of Continuous Point
}

\author{
Haifeng Wang ${ }^{1,}$, Kun Zhang ${ }^{1, b}$, Zhuang $\mathrm{Li}^{1, \mathrm{c}}$ and Hongxu Wang ${ }^{1, \mathrm{~d}}$
}

${ }^{1}$ College of Computer Engineering, Hainan Tropical Ocean University, Sanya, Hainan, 572022, China

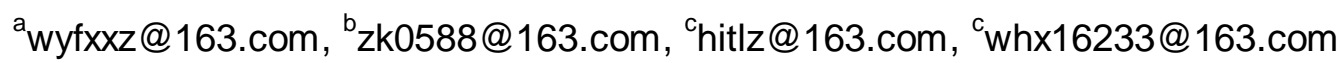

Keywords: Percentage; Continuous point; Inverse fuzzy number; Fuzzy time-series forecasting

\begin{abstract}
Fuzzy time-series forecasting model of inverse fuzzy number based on percentage year to year of continuous point is proposed. We improved the forecasting model of Saxena. The new model puts the percentage year to year of historical data as for the domain of discourse, uses percentage year to year of continuous point to define fuzzy number, and then defines the corresponding inverse fuzzy number. At last we again provide the predictor formula and study the prediction problem of freshman registration number at the University of Alabama, in order to demonstrate the application of a new prediction model. The results show that AFER and MSE of the model are very small compared with the existing models.
\end{abstract}

\section{Introduction}

Zadeh published pioneering thesis fuzzy sets in 1965 which made the research of uncertainty for added a powerful fuzzy set theory. As the time-series prediction model has been applied in many fields by many scholars. For example in literature, data mining is studied. In literature, Stock Market are studied. Because there are many indetermination about prediction problem, the fuzzy set theory is combined with time series that become the new direction for time series prediction model research. Many scholars have done a helpful job. Such as in literature Song first discussed to propose the first-order time-invariant fuzzy time series model and time-variant fuzzy time series model. And the model is applied to the enrollments prediction problem in University of Alabama at Tuscaloosa. Literature respectively put forward improved fuzzy time series forecasting model. Especially the improved model that was proposed by Saxena is applied to the enrollments prediction problem in University of Alabama at Tuscaloosa. The model makes AFER (Average Forecasting Error Rate) and MSE (Mean Square Error) to greatly reduce. This thesis has improved base on the improved model proposed in the literature. Fuzzy time-series forecasting model of inverse fuzzy number based on percentage year by year of continuous point is gained. The model is applied to the enrollments prediction problem in University of Alabama at Tuscaloosa. Compared with other algorithms, the proposed algorithm parameters such as AFER and MSE have been greatly decreased.

\section{Basic Concept}

This section describes some basic concepts that are used in this thesis by literature.

Definition 1 Let $Z=\left\{Z_{1}, Z_{2}, \cdots, Z_{n}\right\}$ be nonempty set, $W$ be named fuzzy subset in $Z$. A map $\mu_{W}: Z \rightarrow[0,1]$ is given from domain of discourse $Z$ to closed interval $[0,1] . \mu_{W}$ is named membership function of fuzzy subset $W . \mu_{W}\left(Z_{i}\right),\left(Z_{i} \in Z, i=1,2, \cdots, n\right)$ is named membership degree of fuzzy set $W$ for the element $Z_{i}$, as follow: $W=\mu_{W}\left(Z_{1}\right) / Z_{1}+\mu_{W}\left(Z_{2}\right) / Z_{2}+\cdots \mu_{W}\left(Z_{n}\right) / Z_{n}$

Definition 2 When the element $Z_{i}(1 \leq i \leq n)$ be real number, fuzzy subset sometimes is named fuzzy number in nonempty set $Z=\left\{Z_{1}, Z_{2}, \cdots, Z_{n}\right\}$. For example when $Z_{i}(1 \leq i \leq n)$ be real number, Triangular fuzzy number in nonempty set $Z=\left\{Z_{1}, Z_{2}, \cdots, Z_{n}\right\}$ can be defined as follows: 


$$
\begin{gathered}
W_{1}=\frac{1}{Z_{1}}+\frac{0.5}{Z_{2}}+\frac{0}{Z_{3}}+\cdots+\frac{0}{Z_{n}} W_{2}=\frac{0.5}{Z_{1}}+\frac{1}{Z_{2}}+\frac{0.5}{Z_{3}}+\frac{0}{Z_{4}}+\cdots+\frac{0}{Z_{n}}, \cdots \\
W_{\beta}=\frac{0}{Z_{1}}+\cdots+\frac{0.5}{Z_{\beta-1}}+\frac{1}{Z_{\beta}}+\frac{0.5}{Z_{\beta+1}}+\cdots+\frac{0}{Z_{n}}, 2 \leq \beta \leq n-1 \quad W_{n}=\frac{0}{Z_{1}}+\cdots+\frac{0}{Z_{n-2}}+\frac{0.5}{Z_{n-1}}+\frac{1}{Z_{n}} .
\end{gathered}
$$

Definition 3 The definition of YYCR (Year to year changes rate) percentage for truthful data is as follow:

$$
Z^{x+i}=\frac{\left(A_{x+i}-A_{x+i-1}\right)}{A_{x+\mathrm{i}-1}} \times 100 \%, i \in\{1,2, \cdots, \mathrm{n}\}
$$

Among $A_{i}, A_{i-1}$ is respectively truthful data in $x+i, x+i-1$ year.

Definition 4 The difference between truthful data $A_{i}$ and predicted data $F_{i}$ is named $F E$ (Forecasting Error), as follow:

$$
F E=A_{i}-F_{i}
$$

Definition 5 Computational formula of MSE is defined as follow:

$$
M S E=\sum_{i=1}^{n}\left(A_{i}-F_{i}\right)^{2} / n
$$

Definition 6 Computational formula of FER (Forecasting Error Rate) is defined as follow:

$$
F E R=\left|A_{i}-F_{i}\right| / A_{i}
$$

Definition 7 Computational formula of AFER is defined as follow:

$$
\text { AFER }=\left(\frac{1}{n} \sum_{i=1}^{n} \frac{\left|A_{i}-F_{i}\right|}{A_{i}}\right)
$$

\section{Suggested New Model}

New model improves the prediction model in. Don't need to set up domain of discourse range firstly. Don't need to request the mid-point of each interval secondly. At last the content of prediction formula for the inverse fuzzy number also is improved bigger. Application steps of the new model are as follows:

Need to study the history of the data list $A_{x+i},(i=x+1, x+2, \cdots, x+n)$;

Using Eq. (1) to calculate the percentage of YYCR for the historical data, $Z^{x+i},(i=1,2, \cdots, n)$;

With the percentage of YYCR for the historical data as the element, theory of discrete domain $Z=\left\{Z^{x+1}, Z^{x+2}, \cdots, Z^{x+n}\right\}$ is established;

Establish the inverse fuzzy number of the continuous point in domain of discourse $Z$ as following:

$$
k_{\beta}= \begin{cases}\frac{1+0.003}{1 / Z_{1}+0.003 / Z_{2}} & , \beta=1 \\ \frac{0.003+1+0.003}{0.003 / Z_{\beta-1}+1 / Z_{\beta}+0.003 / Z_{\beta+1}}, 2 \leq \beta \leq n-1 \\ \frac{0.003+1}{0.003 / Z_{n-1}+1 / Z_{n}} \quad, \beta=n\end{cases}
$$

Set up the new predictor formula (7) as following: 


$$
F_{\beta}=A_{\beta-1} \times\left(1+k_{\beta} \%\right)
$$

Among $A_{\beta-1}$ is historical data in $\beta-1$ year. $F_{\beta}$ is prediction data in $\beta$ year. The formula shows that applying historical data in $\beta$-1year to predict forecast data in $\beta$ year.

Appling prediction formula for historical data to make predictions.

\section{Application Examples of Prediction Research about the Enrollment of the University of Alabama}

A case study of what enrollment prediction problem at the University of Alabama, the improved model will be introduced in this paper. Taking the data of enrollment prediction problem first in literature by Song at the University of Alabama as the research object, the data were listed Table 1.

Table 1 History of enrollment and the percentage of change year by year at the University of Alabama

Table 1

\begin{tabular}{|c|c|c|c|l|c|c|c|c|}
\hline Year & Enrollment & $\begin{array}{c}\text { Year to } \\
\text { year }\end{array}$ & $\begin{array}{c}\text { The } \\
\text { percentage } \\
\text { of change }\end{array}$ & \multicolumn{1}{|c|}{ Element } & $\begin{array}{c}\text { Forecasts } \\
\left(F_{i}\right)\end{array}$ & $A_{i}-F_{i}$ & $\left(A_{i}-F_{i}\right)^{2}$ & $F E R=\frac{\left|A_{i}-F_{i}\right|}{A_{i}}$ \\
\hline 1971 & 13055 & & & & & & & \\
\hline 1972 & 13563 & $1971-72$ & $3.89 \%$ & $Z_{14}^{1972}=3.89$ & 13562 & 1 & 1 & $0.00737 \%$ \\
\hline 1973 & 13867 & $1972-73$ & $2.24 \%$ & $Z_{13}^{1973}=2.24$ & 13867 & 0 & 0 & 0 \\
\hline 1974 & 14696 & $1973-74$ & $5.98 \%$ & $Z_{20}^{1974}=5.98$ & 14697 & -1 & 1 & $0.00680 \%$ \\
\hline 1975 & 15460 & $1974-75$ & $5.20 \%$ & $Z_{16}^{1975}=5.20$ & 15460 & 0 & 0 & 0 \\
\hline 1976 & 15311 & $1975-76$ & $-0.96 \%$ & $Z_{5}^{1976}=-0.96$ & 15300 & 11 & 121 & $0.07184 \%$ \\
\hline 1977 & 15603 & $1976-77$ & $1.91 \%$ & $Z_{12}^{1977}=1.91$ & 15603 & 0 & 0 & 0 \\
\hline 1978 & 15861 & $1977-78$ & $1.65 \%$ & $Z_{10}^{1978}=1.65$ & 15860 & 1 & 1 & $0.00630 \%$ \\
\hline 1979 & 16807 & $1978-79$ & $5.96 \%$ & $Z_{19}^{1979}=5.96$ & 16807 & 0 & 0 & 0 \\
\hline 1980 & 16919 & $1979-80$ & $0.67 \%$ & $Z_{9}^{1980}=0.67$ & 16919 & 0 & 0 & 0 \\
\hline 1981 & 16388 & $1980-81$ & $-3.14 \%$ & $Z_{2}^{1981}=-3.14$ & 16388 & 0 & 0 & 0 \\
\hline 1982 & 15433 & $1981-82$ & $-5.83 \%$ & $Z_{1}^{1982}=-5.83$ & 15435 & -2 & 4 & $0.01296 \%$ \\
\hline 1983 & 15497 & $1982-83$ & $0.41 \%$ & $Z_{8}^{1983}=0.41$ & 15497 & 0 & 0 & 0 \\
\hline 1984 & 15145 & $1983-84$ & $-2.27 \%$ & $Z_{4}^{1984}=-2.27$ & 15146 & -1 & 1 & $0.00660 \%$ \\
\hline 1985 & 15163 & $1984-85$ & $0.12 \%$ & $Z_{7}^{1985}=0.12$ & 15163 & 0 & 0 & 0 \\
\hline 1986 & 15984 & $1985-86$ & $5.41 \%$ & $Z_{17}^{1986}=5.41$ & 15984 & 0 & 0 & 0 \\
\hline 1987 & 16859 & $1986-87$ & $5.47 \%$ & $Z_{18}^{1987}=5.47$ & 16859 & 0 & 0 & 0 \\
\hline 1988 & 18150 & $1987-88$ & $7.66 \%$ & $Z_{21}^{1988}=7.66$ & 18149 & 1 & 1 & $0.00551 \%$ \\
\hline 1989 & 18970 & $1988-89$ & $4.52 \%$ & $Z_{15}^{1989}=4.52$ & 18970 & 0 & 0 & 0 \\
\hline 1990 & 19328 & $1989-90$ & $1.89 \%$ & $Z_{11}^{1990}=1.89$ & 19328 & 0 & 0 & 0 \\
\hline 1991 & 19337 & $1990-91$ & $0.05 \%$ & $Z_{6}^{1991}=0.05$ & 19337 & 0 & 0 & 0 \\
\hline 1992 & 18876 & $1991-92$ & $-2.38 \%$ & $Z_{3}^{1992}=-2.38$ & 18876 & 0 & 0 & 0 \\
\hline AFER & & & & & & & & $0.00559 \%$ \\
\hline MSE & & & & & & & 130 & \\
\hline & & & & & & 0 & 0 \\
\hline
\end{tabular}

Using Eq. (1) to Calculate the Percentage of YYCR for the Historical Data. In literature, Saxena has calculated the percentage of YYCR for enrollment at the University of Alabama in 1971-1992.In this case, let $x=1971$, using Eq. (1) to calculate. 


$$
Z^{1972}=\left(A_{1972}-A_{1971}\right) / A_{1971}=(13563-13055) / 13055=3.89 \% \text {. }
$$

With the Percentage of YYCR for the Historical Data as the Element, Theory of Discrete Domain is Established. The elements of domain are the percentage of the historical data YYCR by size. Theory of discrete domain that is formed by the percentage of enrollment YYCR at the University of Alabama in 1971-1992 as follows:

$$
\begin{aligned}
Z=\{ & Z_{1}^{1982}=-5.83, Z_{2}^{1981}=-3.14, Z_{3}^{1992}=-2.38, Z_{4}^{1984}=-2.27, Z_{5}^{1976}=-0.96, Z_{6}^{1991}=0.05, Z_{7}^{1985}=0.12, \\
& Z_{8}^{1983}=0.41, Z_{9}^{1980}=0.67, Z_{10}^{1978}=1.65, Z_{11}^{1990}=1.89, Z_{12}^{1977}=1.91, Z_{13}^{1973}=2.24, Z_{14}^{1972}=3.89, \\
& \left.Z_{15}^{1989}=4.52, Z_{16}^{1975}=5.20, Z_{17}^{1986}=5.41, Z_{18}^{1987}=5.47, Z_{19}^{1979}=5.96, Z_{20}^{1974}=5.98, Z_{21}^{1988}=7.66\right\}
\end{aligned}
$$

Among subscript of the elements indicate their serial number. Superscripts of the elements indicate the year of the elements. For example $Z_{2}^{1981}=-3.14$, it shows that the serial number of the element is 2 and the year of the element is 1981. In order to calculate conveniently, Eq. (8) is rewritten into domain of discourse that superscript in chronological order and the subscript is still according to the serial number of the elements. Domain of discourse is as follows:

$$
\begin{gathered}
Z=\left\{Z_{14}^{1972}=3.89, Z_{13}^{1973}=2.24, Z_{20}^{1974}=5.98, Z_{16}^{1975}=5.20, Z_{5}^{1976}=-0.96, Z_{12}^{1977}=1.91, Z_{10}^{1978}=1.65,\right. \\
Z_{19}^{1979}=5.96, Z_{9}^{1980}=0.67, Z_{2}^{1981}=-3.14, Z_{1}^{1982}=-5.83, Z_{8}^{1983}=0.41, Z_{4}^{1984}=-2.27, Z_{7}^{1985}=0.12, \\
\left.Z_{17}^{1986}=5.41, Z_{18}^{1987}=5.47, Z_{21}^{1988}=7.66, Z_{15}^{1989}=4.52, Z_{11}^{1990}=1.89, Z_{6}^{1991}=0.05, Z_{3}^{1992}=-2.38\right\}
\end{gathered}
$$

Application of Prediction Formula for Historical Data to Make Predictions. Applying the inverse fuzzy number Eq. (6) which fuzzy number of normal distribution and the prediction Eq. (7), enrollment is simulated prediction in 1971-1992 at the University of Alabama, among $\mathrm{n}=21$. For example:

$$
\begin{aligned}
& k_{1972}=\frac{0.003+1+0.003}{\frac{0.003}{Z_{13}^{1973}}+\frac{1}{Z_{14}^{1972}}+\frac{0.003}{Z_{15}^{1989}}=\frac{1.006}{\frac{0.003}{2.24}+\frac{1}{3.89}+\frac{0.003}{4.52}}=3.883090} \\
& F_{1972}=F_{1971} \times\left(1+k_{1972} \%\right)=13055 \times(1+3.883090 \%)=13562 \\
& A_{1972}-F_{1972}=13563-13562=1,\left|A_{1972}-F_{1972}\right| / A_{1972}=1 \div 13563=0.000074
\end{aligned}
$$

The computed data are filled in Table 1 too.

\section{The Comparison of Different Forecasting Models}

Applying the different models that were proposed in this paper and literature, we come into studying enrollment prediction at the University of Alabama. The given results are listed in Table 2. If use AFER and MSE of these two indexes to evaluate the merits of the forecasting model, the forecasting model that proposed by Saxena in literature and presented in this paper are all better.

\section{Conclusion}

For more than 20 years, people unremitting efforts, the original prediction model is continuously improved, and new ideas emerge continuously. For the application at the University of Alabama in enrollment prediction problem, AFER and MSE of the proposed model in this paper are less than the existing methods. Combining calendar year enrollment plan, these studies will make people having a more detailed understanding for history change law about enrollment at the University of Alabama. To study and analysis the reasons of new student erosion and formulate the new recruitment plan that it is good. Developing fuzzy time series prediction method of new higher prediction accuracy, especially developing fuzzy time series forecasting model to predict the unknown year data and expanding application field of fuzzy time series forecasting model that should be a research direction of fuzzy time series forecasting method. 
Table 2 Comparison of Different Forecasting Models

\begin{tabular}{|c|c|c|c|c|c|c|c|c|c|c|c|}
\hline Year & Enrollments & Song & Porter & Jilani & Chen & Hwang & Jilani & Jilani & Huarng & Saxena & Proposed \\
\hline 1971 & 13055 & - & - & 13579 & - & - & - & 14464 & - & - & - \\
\hline 1972 & 13563 & & 13410 & 13798 & - & - & - & 14464 & 14000 & 13486 & 13562 \\
\hline 1973 & 13867 & & 13932 & 13798 & - & - & - & 14464 & 14000 & 13896 & 13867 \\
\hline 1974 & 14696 & & 14664 & 14452 & 14500 & - & 14730 & 14710 & 14000 & 14698 & 14697 \\
\hline 1975 & 15460 & 14700 & 15423 & 15373 & 15500 & - & 15615 & 15606 & 15500 & 15454 & 15460 \\
\hline 1976 & 15311 & 14800 & 15847 & 15373 & 15500 & 16260 & 15614 & 15606 & 15500 & 15595 & 15300 \\
\hline 1977 & 15603 & 15400 & 15580 & 15623 & 15500 & 15511 & 15611 & 15606 & 16000 & 15600 & 15603 \\
\hline 1978 & 15861 & 15500 & 15877 & 15883 & 15500 & 16003 & 15611 & 15606 & 16000 & 15844 & 15860 \\
\hline 1979 & 16807 & 15500 & 16773 & 17079 & 16500 & 16261 & 16484 & 16470 & 16000 & 16811 & 16807 \\
\hline 1980 & 16919 & 16800 & 16897 & 17079 & 16500 & 17407 & 16476 & 16470 & 17500 & 16916 & 16919 \\
\hline 1981 & 16388 & 16200 & 16341 & 16497 & 16500 & 17119 & 16469 & 16470 & 16000 & 16425 & 16388 \\
\hline 1982 & 15433 & 16400 & 15671 & 15373 & 15500 & 16188 & 15609 & 15606 & 16000 & 15657 & 15435 \\
\hline 1983 & 15497 & 16800 & 15507 & 15373 & 15500 & 14833 & 15614 & 15606 & 15500 & 15480 & 15497 \\
\hline 1984 & 15145 & 16400 & 15200 & 15024 & 15500 & 15497 & 15612 & 15606 & 16000 & 15214 & 15146 \\
\hline 1985 & 15163 & 15500 & 15218 & 15024 & 15500 & 14745 & 15609 & 15606 & 16000 & 15184 & 15163 \\
\hline 1986 & 15984 & 15500 & 16035 & 15883 & 15500 & 15163 & 15606 & 15606 & 16000 & 15995 & 15984 \\
\hline 1987 & 16859 & 15500 & 16903 & 17079 & 16500 & 16384 & 16477 & 16470 & 16000 & 16861 & 16859 \\
\hline 1988 & 18150 & 16800 & 17953 & 17991 & 18500 & 17659 & 18482 & 18473 & 17500 & 17965 & 18149 \\
\hline 1989 & 18970 & 19300 & 18879 & 18802 & 18500 & 19150 & 18481 & 18473 & 19000 & 18964 & 18970 \\
\hline 1990 & 19328 & 17800 & 19303 & 18994 & 19500 & 19770 & 19158 & 19155 & 19000 & 19329 & 19328 \\
\hline 1991 & 19337 & 19300 & 19432 & 18994 & 19500 & 19928 & 19155 & 19155 & 19500 & 19378 & 19337 \\
\hline 1992 & 18876 & 19600 & 18966 & 18916 & 18500 & 15837 & 18475 & 18473 & 19000 & 18984 & 18876 \\
\hline AFER & $3.22 \%$ & $3.11 \%$ & $0.57 \%$ & $1.02 \%$ & $1.52 \%$ & $2.44 \%$ & $1.40 \%$ & $2.39 \%$ & $1.53 \%$ & $0.34 \%$ & $0.00559 \%$ \\
\hline MSE & 423027 & 407507 & 21575 & 41426 & 86696 & 226611 & 82269 & 227194 & 86694 & 9169 & 130 \\
\hline
\end{tabular}

\section{Acknowledgements}

This work was financially supported by the key project of scientific research of Hainan Province(No.Hnky2015ZD-14), the scientific and technological cooperative project for college and region of Sanya (2015YD16, 2014YD30), the key Laboratory of Sanya Project(No.L1410).

\section{References}

[1] Zadeh L A: Fuzzy sets. Information and Control, Vol.8 (1965), pp. 338-353.

[2] Chen C-H, Hong T-P: Tseng V S. fuzzy data mining for time-series data. Applied Soft Computing, vol.12 (2012), pp. 536-542.

[3] Chu H-H, Chen T-L, Cheng C-H and Huang C-C: Fuzzy dual-factor time-service for stock index forecasting. Expert Systems with Application, vol.36 (2009), pp. 165-171.

[4] Song Q, Chissom B S: Fuzzy time series and its models. Fuzzy Set and Systems, vol. 54 (1993), pp.269-277.

[5] Song Q, Chissom B S: Forecasting enrollments with fuzzy time series--part 1. Fuzzy Set and Systems, vol. 54 (1993), pp .1-9.

[6] S M Chen: Forecasting enrollments based on high-order fuzzy time series. Cybernetics and Systems: An International Journal, 2002, vol. 33. pp. 1-16.

[7] J R Hwang, S M Chen, C H Lee: Handling forecasting problems using fuzzy time series. Fuzzy Sets and Systems, 1998, vol.100, pp. 217-228.

[8] Stevension Porter: Fuzzy time series forecasting using percentage change as the universe of discourse. Proceedings of World Academy of Science. Engineering and Technology. vol.55(2009), pp.154-157 
[9] Tahseen Ahmed Jilani, Syed Muhammad Aqil Burney, and Cemal Ardil: Fuzzy metric approach for fuzzy time series forecasting based on frequency density based partitioning. World Academy of Science, Engineering and Technology, Vol. 34(2007), pp. 1-6.

[10] Preetika Saxena, Kalyani Sharma and Santhosh Easo: Foreca enrollment based on fuzzy time series with higher forecast accuracy rate. International Journal of Computer Technology and Applications, vol. 3. No. 3(2012).pp.957-961.

[11] T A Jilani, S M A Burney: M-factor high order fuzzy time series forecasting for road accident data. Analysis and design of intelligent systems using soft computing techniques. Springer Berlin Heidelberg, (2007).p.246-254.

[12] T A Jilani, S M A Burney and C Ardil: Multivariate high order fuzzy time series forecasting for car road accidents. International Journal of Computational Intelligence, vol. 4, no. 1(2007), pp. $15-20$

[13] K Huarng: Heuristic models of fuzzy time series for forecasting. Fuzzy Sets and Systems, Vol. 123(2001), pp.369-386. 